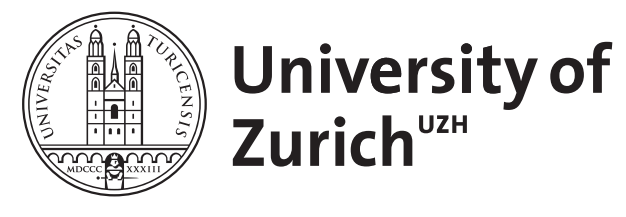

\title{
Spin-lattice relaxation in cuprate superconductors
}

\author{
Brinkmann, D
}

\begin{abstract}
This presentation gives a personal review of nuclear magnetic resonance (NMR) and nuclear quadrupole resonance (NQR) spin-lattice relaxation studies in cuprate superconductors mainly dealing with the $\mathrm{YBa} 2 \mathrm{Cu} 4 \mathrm{O} 8$ compound with many examples from the Zürich laboratory. The studies were performed in both the normal and the superconducting state with various NMR isotopes (e.g.,17O,63,65Cu,135,137Ba). The relatively broad signals were mostly obtained by a phase-alternating add-subtract spin-echo technique. We will discuss the general behavior of spin-lattice relaxation in the normal state and the calculation of the dynamic spin including an approach (on the basis of thet-J model) to calculate the relaxation for plane copper, oxygen, and yttrium. An application of the Luttingerliquid model to the relaxation of chain copper in $\mathrm{YBa} 2 \mathrm{Cu} 3 \mathrm{O} 7$ and $\mathrm{YBa} 2 \mathrm{Cu} 4 \mathrm{O} 8$ is also given. We then will deal with characteristic features of the $\mathrm{YBa} 2 \mathrm{Cu} 4 \mathrm{O} 8$ structure: the spin gap, an electronic crossover in the normal state, the single-spin fluid model, and the d-wave pairing
\end{abstract}

DOI: https://doi.org/10.1007/bf03166315

Posted at the Zurich Open Repository and Archive, University of Zurich

ZORA URL: https://doi.org/10.5167/uzh-156245

Journal Article

Published Version

Originally published at:

Brinkmann, D (2004). Spin-lattice relaxation in cuprate superconductors. Applied Magnetic Resonance, 27(1-2):207-213.

DOI: https://doi.org/10.1007/bf03166315 


\title{
Spin-Lattice Relaxation in Cuprate Superconductors
}

\author{
D. Brinkmann \\ Physik-Institut, University of Zürich, Zürich, Switzerland
}

Received September 10, 2003

\begin{abstract}
This presentation gives a personal review of nuclear magnetic resonance (NMR) and nuclear quadrupole resonance (NQR) spin-lattice relaxation studies in cuprate superconductors mainly dealing with the $\mathrm{YBa}_{2} \mathrm{Cu}_{4} \mathrm{O}_{8}$ compound with many examples from the Zürich laboratory. The studies were performed in both the normal and the superconducting state with various NMR isotopes (e.g., ${ }^{17} \mathrm{O}$, ${ }^{63,65} \mathrm{Cu},{ }^{135,137} \mathrm{Ba}$ ). The relatively broad signals were mostly obtained by a phase-alternating add-subtract spin-echo technique. We will discuss the general behavior of spin-lattice relaxation in the normal state and the calculation of the dynamic spin including an approach (on the basis of the $t-J$ model) to calculate the relaxation for plane copper, oxygen, and yttrium. An application of the Luttingerliquid model to the relaxation of chain copper in $\mathrm{YBa}_{2} \mathrm{Cu}_{3} \mathrm{O}_{7}$ and $\mathrm{YBa}_{2} \mathrm{Cu}_{4} \mathrm{O}_{8}$ is also given. We then will deal with characteristic features of the $\mathrm{YBa}_{2} \mathrm{Cu}_{4} \mathrm{O}_{8}$ structure: the spin gap, an electronic crossover in the normal state, the single-spin fluid model, and the d-wave pairing.
\end{abstract}

\section{Introduction}

The nuclear magnetic resonance (NMR) and nuclear quadrupole resonance (NQR) signals obtained from cuprate high-temperature superconductors like $\mathrm{YBa}_{2} \mathrm{Cu}_{3} \mathrm{O}_{7}$ and $\mathrm{YBa}_{2} \mathrm{Cu}_{4} \mathrm{O}_{8}$ are very broad depending on the isotope studied and the preparation of the material. One therefore employs, in most cases, the NQR spin-echo method in zero magnetic field with standard pulsed spectrometers, and the signals are obtained, for instance, by a phase-alternating add-subtract spin-echo technique. This way, it has been possible to collect a wealth of information on NMR and NQR parameters like electric field gradients (EFG), Knight shifts, and various types of relaxation times, especially the spin-lattice relaxation time.

This presentation will give a personal review of such relaxation studies mainly dealing with the $\mathrm{YBa}_{2} \mathrm{Cu}_{4} \mathrm{O}_{8}$ compound, known for its homogeneity and stability, with many examples from the Zürich laboratory. The studies were performed in both the normal and the superconducting state with various NMR isotopes (e.g., ${ }^{17} \mathrm{O},{ }^{63,65} \mathrm{Cu},{ }^{135,137} \mathrm{Ba}$ ). One obtains information about the type of coupling between the nucleus and its surrounding (i.e., magnetic or quadrupole coupling) and about the mechanism causing the fluctuations (e.g., phonons, polarons, anti- 
ferromagnetic [AF] fluctuations). The results then provide insight into the microscopic dynamics of the compounds and may help to understand the superconducting phenomenon. References $1-5$ are a selection of general reviews of NMR-NQR studies in high-temperature superconductors.

We will start by discussing the general behavior of spin-lattice relaxation in the normal state and the calculation of the dynamic spin susceptibility. Then, an approach will be discussed, based on the presentation of the $t-J$ model in terms of Hubbard operators, which allows one to calculate the relaxation for plane copper, oxygen, and yttrium. An application of the Luttinger-liquid model to the relaxation of chain copper in $\mathrm{YBa}_{2} \mathrm{Cu}_{3} \mathrm{O}_{7}$ and $\mathrm{YBa}_{2} \mathrm{Cu}_{4} \mathrm{O}_{8}$ is also given. We then will deal with characteristic features of the $\mathrm{YBa}_{2} \mathrm{Cu}_{4} \mathrm{O}_{8}$ structure: the spin gap, an electronic crossover in the normal state, the single-spin fluid model, a separation of charge and spin excitations, and the d-wave pairing. We will show how the various results can be interpreted by models typical for these substances which are derived from AF parent compounds.

\section{Relaxation in the Normal State and the Dynamic Spin Susceptibility}

The fluctuating part of the hyperfine interactions of the nuclear magnetic moments is the source of relaxation processes. The main contribution to the copper, oxygen, and yttrium spin-lattice relaxation rate $1 / T_{1}$ in cuprate compounds arises from electron spin fluctuations, although the presence of quadrupole interactions may have important consequences, as we will see later. The magnetic contribution is related to the imaginary part of the dynamical spin susceptibility $\chi\left(\mathbf{q}, \omega_{0}\right)$ dependent on the wavevector $\mathbf{q}$ and it is given by the Moriya formula

$$
\left(\frac{1}{T_{1} T}\right)_{\alpha}=\frac{\gamma_{\mathrm{n}}^{2} k_{\mathrm{B}}}{2 \mu_{\mathrm{B}}^{2}} \sum_{\mathbf{q}, \alpha^{\prime} \neq \alpha} F_{\alpha^{\prime}}(\mathbf{q}) \frac{\chi_{\alpha^{\prime} \alpha^{\prime}}^{\prime \prime}\left(\mathbf{q}, \omega_{0}\right)}{\omega_{0}},
$$

where

$$
F_{\alpha}(\mathbf{q})=\left|\sum_{j} A_{j, \alpha \alpha} \exp \left(\mathrm{iq} \cdot \mathbf{r}_{j}\right)\right|^{2} .
$$

Here, $F_{\alpha}(\mathbf{q})$ is the form factor, $\omega_{0}$ is the nuclear resonance frequency, $\alpha$ denotes the direction of quantization, i.e., the direction of either the maximum component of the EFG in NQR or of the steady magnetic field in NMR, and $\alpha^{\prime}$ is the direction perpendicular to $\alpha . \mathbf{A}_{j}$ is the on-site $\left(\mathbf{r}_{j}=0\right)$ and transferred $\left(\mathbf{r}_{j} \neq 0\right)$ hyperfine coupling tensor for the nuclei under consideration. Therefore, $1 / T_{1} T$ provides information about the q-averaged imaginary part of $\chi(q, 0)$. The numerical values of the coupling tensors $\mathbf{A}_{j}$, which may range up to several kilooersted, have been derived from various NMR experiments, in particular from magnetic shift studies. 
Relaxation times and Knight shift behave quite differently from corresponding data in conventional superconductors or metals. For instance, the relaxation rate has a nonlinear temperature dependence and the Knight shift is temperaturedependent. Furthermore, different nuclei $(\mathrm{Cu}, \mathrm{O}, \mathrm{Y})$ display different temperature dependences which, in turn, are related to the doping level of the substance. For instance, $1 / T_{1} T$ of $\mathrm{Cu}(2)$ in the normal state of $\mathrm{YBa}_{2} \mathrm{Cu}_{4} \mathrm{O}_{8}$ increases with decreasing temperature in a Curie-Weiss-like fashion, reaches a maximum at a temperature $T^{*}$, which characterizes the spin-gap (to be discussed later), and then continuously diminishes as the temperature goes to zero.

One of the first attempts to explain the temperature dependence of $1 / T_{1}$ was the phenomenological MMP model by Millis et al. [6]. The essential assumption of this model is that $\chi\left(\mathbf{q}, \omega_{0}\right)$ is the superposition of two terms: one for itinerant quasiparticles and the other for localized $\mathrm{Cu}^{2+}$ magnetic moments. Together with its variants, the model has, among others, quantitatively described, for example, the normal state relaxation of $\mathrm{Cu}, \mathrm{O}$ and $\mathrm{Y}$ in $\mathrm{YBa}_{2} \mathrm{Cu}_{3} \mathrm{O}_{7}$ and $\mathrm{La}_{1.85} \mathrm{Sr}_{0.15} \mathrm{CuO}_{4}$. Further developments of the MPP model and the so-called nearly AF Fermi liquid (NAFL) description of the superconductors are reviewed in ref. 7. References 4 and 5 discuss these models and alternatives like the 2DQHAF scaling model which regards a cuprate superconductor as a disordered two-dimensional (2-D) quantum Heisenberg AF. It seems that the NAFL model is rather well obeyed in overdoped cuprates, while the 2-DQHAF model describes underdoped cuprates.

The $t-J$ model [8] is a good starting point for calculations of the dynamic spin susceptibility, at least for compounds with moderate doping. Here, $t$ denotes the hopping energy of the holes and $J$ represents the strong repulsion between holes residing on the same square. When calculating the susceptibility, an essential point is how one is treating the AF correlation length $\xi$ which depends on temperature and doping. Is the dependence postulated? Is it obtained from a comparison with experimental data? Expressions used for $\chi\left(\mathbf{q}, \omega_{0}\right)$ are good approximations only for wavevectors in the vicinity of the $\mathrm{AF}$ wavevector; thus, the models provide reliable results for $1 / T_{1}$ if $\xi$ is large. However, in $\mathrm{YBa}_{2} \mathrm{Cu}_{3} \mathrm{O}_{7}$, for instance, $\xi$ is small and therefore a microscopic approach is required.

As an example, we have calculated $\chi^{\prime \prime}\left(\mathbf{q}, \omega_{0}\right)$ within a constraint-free theory based on the presentation of the $t-J$ model in terms of Hubbard operators [9]. Both electron and AF spin correlation were taken into account. The model is able to reproduce the main features of the temperature and doping dependence of $\xi$ in both the pure Heisenberg $\mathrm{AF}$ (e.g., $\mathrm{La}_{2} \mathrm{CuO}_{4}$ ) and doped compounds $\mathrm{La}_{2-x} \mathrm{Sr}_{x} \mathrm{CuO}_{4}$. We then determined $1 / T_{1}$ for ${ }^{17} \mathrm{O},{ }^{63} \mathrm{Cu}$, and ${ }^{89} \mathrm{Y}$ in $\mathrm{YBa}_{2} \mathrm{Cu}_{3} \mathrm{O}_{7}$; the results are in good agreement with experimental data.

The relaxation of chain copper in $\mathrm{YBa}_{2} \mathrm{Cu}_{3} \mathrm{O}_{7}$ and $\mathrm{YBa}_{2} \mathrm{Cu}_{4} \mathrm{O}_{8}$ requires a different treatment [10] since the chains present an 1-D quantum system. It is believed that the Luttinger-liquid approach is most appropriate for describing the universal low-energy properties of such systems and the interactions between their elementary excitations. Our starting point for describing the charge and spin dynamics in the chains of cuprate superconductors is the 1-D Hubbard model, 
whose low-energy properties can be described within the Luttinger-liquid approach. On this basis, we calculated the temperature and concentration dependence of $1 / T_{1}$ and the Knight shift of the chain $\mathrm{Cu}$ nuclei in $\mathrm{YBa}_{2} \mathrm{Cu}_{3} \mathrm{O}_{7}$ and $\mathrm{YBa}_{2} \mathrm{Cu}_{4} \mathrm{O}_{8}$. The experimental results are well fitted by the predictions of the Luttinger-Tomonaga model and the renormalization group theory. The fit yields parameters which are reasonable for both compounds.

\section{Spin Gap and Electronic Crossover}

The occurrence of the so-called pseudogap is a characteristic feature of the normal state of optimally doped and underdoped cuprate superconductors. The pseudogap refers to the transfer of low-energy excited states to higher energy. In NMR and neutron experiments, the pseudogap reveals itself as a spin gap. We have studied the spin gap in $\mathrm{YBa}_{2} \mathrm{Cu}_{4} \mathrm{O}_{8}$, where $1 / T_{1} T$ reaches a maximum at $T^{*}$, about 150 $\mathrm{K}$. Is it possible that the spin gap is related to other gaps present in the superconductor?

During the course of our spin gap investigations, we discovered anomalies (like kinks and change of slope) in the temperature behavior of several NMRNQR quantities, all occurring around a temperature $T^{+}=180 \mathrm{~K}$ [11]. These anomalies were found in spin-lattice relaxation rates of $\mathrm{Cu}, \mathrm{O}$, and $\mathrm{Y}$ but also in line widths and Knight shifts. We interpreted the anomalies as the fingerprint of an electronic crossover, rather than a structural phase transition, occurring in both the planes and chains once the temperature is lowered below $T^{+}$. The crossover reveals itself by enhanced charge fluctuations in planes and chains accompanied by a charge (hole) transfer from chain to plane. Anomalies around $\mathrm{T}^{+}$ were also observed in Raman scattering and X-ray diffraction [11].

These anomalies seem to support the idea that the spin gap phenomenon is caused, at least partly, by a transition due to a charge density wave (CDW), and hence, that the spin gap and electronic crossover are related. Eremin et al. [12] have calculated the temperature dependence of the spin susceptibility in $\mathrm{YBa}_{2} \mathrm{Cu}_{4} \mathrm{O}_{8}$ on the assumption that a pseudogap in the normal state opens due to a CDW instability. The agreement with experiment is very good. The authors explained, for example, the strong temperature dependence of the magnetic shift of $\mathrm{Cu}(2)$ nuclei and predicted a dependence of $T^{*}$ on the isotope mass.

Such an isotope effect has been found in a high-accuracy NQR spin-lattice relaxation study of the plane ${ }^{63} \mathrm{Cu}$ nuclei, supplemented by susceptibility measurements, on ${ }^{16} \mathrm{O}$ - and ${ }^{18} \mathrm{O}$-exchanged $\mathrm{YBa}_{2} \mathrm{Cu}_{4} \mathrm{O}_{8}$ samples [13]. The isotope exponents, defined as $\alpha=-\Delta \ln \left(T^{*}\right) / \Delta \ln (m)$ for $T^{*}$, and correspondingly for $T_{c}$, are $\alpha\left(T^{*}\right)=$ $0.061(8)$ and $\alpha\left(T_{\mathrm{c}}\right)=0.056(12)$. The agreement of the exponents, within the experimental error, suggests a common origin, or at least a relation, for the superconducting and the spin gap. By extending their work to the plane $\mathrm{Cu}$ Knight shift, Mali et al. [14] again found an isotope effect on the spin gap; both exponents have the same sign. These results disagree with a study which reported the absence of an isotope effect [15]. Hence, the controversy on this topic continues. 


\section{Quadrupole Relaxation of Barium Nuclei}

While the AF spin fluctuations dominate the plane $\mathrm{Cu}$ and $\mathrm{O}$ nuclear relaxation, they have a negligible effect on the spin-lattice relaxation of the out-of-plane $\mathrm{Ba}$ nuclei, ${ }^{135} \mathrm{Ba}$ and ${ }^{137} \mathrm{Ba}$. Instead, these nuclei relax by the interaction of their quadrupole moments, $e Q$, with fluctuating EFG due to phonons [16]. It turned out that the $\mathrm{Ba}$ relaxation is caused by a two-phonon Raman process, i.e., the absorption of one phonon and the emission of another. On the assumption of a Debye type phonon spectrum, such a process leads to the following relaxation rate

$$
\frac{1}{T_{1}}=A Q^{2} \int_{0}^{k T_{\mathrm{D}} / \hbar} \frac{\exp (\hbar \omega / k T)}{(\exp (\hbar \omega / k T)-1)^{2}}\left(\frac{\hbar \omega}{k T_{\mathrm{D}}}\right)^{6} \mathrm{~d} \omega .
$$

Here, $T_{\mathrm{D}}$ is the Debye temperature and the factor $A$ is assumed to be temperature independent. This expression has been fitted to the experimental data of both isotopes with $T_{\mathrm{D}}$ as the only adjustable parameter, beside the scaling factor $A$. The fit is very good for both isotopes and yields the same Debye temperature, $T_{\mathrm{D}}=185(20) \mathrm{K}$. In the high- and low-temperature limits, the relaxation rates vary as $T^{2}$ and $T^{\dagger}$, respectively, which is well known from relaxation studies of ionic crystals. It is worthwhile to note that the $\mathrm{Ba}$ relaxation rates pass smoothly "through $T_{\mathrm{c}}$ ", which implies that those phonons causing Ba relaxation do not change appreciably at the phase transition

Our result for the Debye temperature agrees quite well with $T_{\mathrm{D}}$ values of 155$185 \mathrm{~K}$ deduced from an analysis of the thermal conductivity. This is expected since heat is mostly carried by acoustical phonons and the Debye model, which was used to derive the relaxation formula, works best for acoustical phonons.

For $\mathrm{YBa}_{2} \mathrm{Cu}_{3} \mathrm{O}_{7}$, it has been reported that magnetic interaction seems to be the origin of the $\mathrm{Ba}$ relaxation [17]. The origin of the very different behavior of $\mathrm{Ba}$ relaxation in the two cuprate compounds is unknown. It is feasible that oxygen diffusion in $\mathrm{YBa}_{2} \mathrm{Cu}_{3} \mathrm{O}_{7}$ accounts for the difference.

\section{Single-Spin Fluid Model}

After this digression, we return to the plane $\mathrm{Cu}$ and $\mathrm{O}$ relaxation in the normal state of $\mathrm{YBa}_{2} \mathrm{Cu}_{4} \mathrm{O}_{8}$ and we will show that even here quadrupole relaxation is present. These investigations are related to the question whether the single-spin fluid (SSF) model is valid, in other words: How many electronic degrees of freedom are necessary to describe the physics at the atomic scale of cuprate superconductors? Adopting the picture of the spin-resonant singlet state with quasi-localized holes at copper [5], only one spin degree of freedom would be necessary; and copper and oxygen Knight shift data seem to favor this view. However, oxygen and yttrium relaxation anisotropy data do not fit into this picture.

First, the anisotropy of the in-plane oxygen spin-lattice relaxation rate, ${ }^{17}\left(W_{\|}, W_{\mathrm{c}}\right)$ (with the magnetic field either in the plane or parallel to the $c$-axis), displays a 
pronounced temperature dependence (e.g., [18]) while the SSF model predicts an almost temperature-independent rate $\left(W\right.$ is an abbreviation for $\left.1 / T_{1}\right)$. Zha et al. [19] tried to explain the behavior of ${ }^{17}\left(W_{\|}, W_{\mathrm{c}, \perp}\right)$ in the framework of the MPP by including additional next-nearest-neighbor and incommensurate AF fluctuations. This model is able to describe the temperature dependence of ${ }^{17}\left(W_{\|}, W_{c, 1}\right)$ quite well. However, when calculating the ratio ${ }^{17} W_{\mathrm{c}}{ }^{89} W_{\mathrm{c}}$ by following the MPP model and including the new form factor proposed by $\mathrm{Zha}$ as well as the incommensurability, our result failed to reproduce the data. While the nearly constant value of ${ }^{17} W_{\mathrm{c}} /{ }^{89} W_{\mathrm{c}}$ at temperatures above $T^{*}$ agrees with the calculation, the pronounced upturn of the ratio below $T^{*}$ and its saturation around $100 \mathrm{~K}$ are not reproduced by the model.

As an alternative, we proposed $[18,20]$ the presence of an additional relaxation mechanism which can provide a coherent description of the temperature dependence of both ${ }^{17}\left(W_{\|}, W_{\mathrm{c}, L}\right)$ and ${ }^{17} W_{\mathrm{c}} /{ }^{89} W_{\mathrm{c}}$. As shown in ref. 18 , there are enhanced charge fluctuations in the chain and the plane at temperatures below $T^{*}$. These fluctuations open an additional relaxation channel which is of quadrupolar origin. This would easily explain the temperature dependence of ${ }^{17} W_{\mathrm{c}} /{ }^{89} \mathrm{~W}_{\mathrm{c}}$ since only ${ }^{17} W_{\mathrm{c}}$ of plane $\mathrm{O}$ is enhanced while $\mathrm{Y}$, because of its spin $1 / 2$, cannot couple to the charge fluctuations.

We employed a double irradiation method [21] to show that, below around $200 \mathrm{~K}$, the spin-lattice relaxation rate of plane oxygen is driven not only by magnetic but also significantly by quadrupolar fluctuations, i.e., low-frequency charge fluctuations. These findings show that, with the opening of the pseudospin gap, a charge degree of freedom is present in the electronic low-energy excitation spectrum. In other words: the SSF model is only partially correct. Two degrees of freedom are involved in the system, one of them is the single-spin degree, whereas the other one is the charge degree of freedom with predominantly oxygen character, since it is not observed at the copper sites.

\section{Superconducting State}

The nature of the nuclear spin-lattice relaxation in the superconducting phase of the cuprate high-temperature superconductors is still a matter of debate. At temperatures above about $0.4 T_{c}$, the plane $\mathrm{Cu}$ relaxation arises from a magnetic interaction of the nuclei with their surroundings. The temperature dependence of the relaxation rate is close to a $T^{3}$ behavior which is attributed to the d-wave pairing of the charge carriers. For a discussion, see e.g., refs. 5 and 7. At low temperatures, however, there are deviations from the $T^{3}$ relation; and at least in some cases, quadrupole interactions are involved. For instance, Kitaoka et al. [22] measured the $\mathrm{Cu}$ relaxation rate in $\mathrm{YBa}_{2} \mathrm{Cu}_{3} \mathrm{O}_{7}$ (with $T_{\mathrm{c}}=90 \mathrm{~K}$ ) and found that the rate becomes temperature-independent below $4.2 \mathrm{~K}$, where it is dominated by quadrupole interactions. Also Takigawa et al. [23] detected, in the same compound, a quadrupole contribution to the $\mathrm{Cu}$ relaxation below $10 \mathrm{~K}$; the authors attributed this contribution to oxygen diffusion. 
A systematic study of the low-temperature plane $\mathrm{Cu}$ relaxation in $\mathrm{YBa}_{2} \mathrm{Cu}_{4} \mathrm{O}_{8}$, where oxygen diffusion is absent, has been reported only quite recently [24]. Between 15 and $1 \mathrm{~K}$, the relaxation rate decreases on lowering the temperature, the rate reaches a minimum at $7 \mathrm{~K}$, and then increases. Such a kind of behavior has not been observed so far in high-temperature superconductors. The total rate consists of a magnetic and a quadrupole contribution. The latter increases with falling temperature, while the magnetic contribution decreases. A study of this unusual behavior is in progress.

\section{References}

1. Pennington C.H., Slichter C.P. in: Physical Properties of High Temperature Superconductors (Ginsburg D.M., ed.), vol. 2, pp. 269-367. Singapore: World Scientific 1990.

2. Mehring M. (ed.): Appl. Magn. Reson. 3, 383-747 (1992)

3. Brinkmann D., Mali M. in: NMR - Basic Principles and Progress (Diehl P., Fluck E., Guenther H., Kosfeld R., Seelig J., eds.), vol. 31, pp. 171-211. Berlin, Heidelberg: Springer 1994.

4. Berthier C., Julien M.H., Horvatic M., Berthier Y.: J. Phys. I 6, 2205-2236 (1996)

5. Rigamonti A., Borsa F., Carretta P.: Rep. Prog. Phys. 61, 1367-1439 (1998)

6. Millis A.J., Monien H., Pines D.: Phys. Rev. B 42, 167-178 (1990)

7. Pines D.: Z. Phys. 103, 129-135 (1997)

8. Zhang F.C., Rice T.M.: Phys. Rev. B 37, 3759 (1988)

9. Zavidonov A.Yu., Brinkmann D.: Phys. Rev. B 63, 132506-1 (2001)

10. Zavidonov A.Yu., Brinkmann D.: Phys. Rev. B 61, 3282-5 (2000)

11. Suter A., Mali M., Roos J., Brinkmann D., Karpinski J., Kaldis E.: Phys. Rev. B 56, 5542-5551 (1997)

12. Eremin I., Eremin M., Varlamov S., Brinkmann D., Mali M., Roos J.: Phys. Rev. B 56, 1130511311 (1997)

13. Raffa F., Ohno T., Mali M., Roos J., Brinkmann D., Condor K., Eremin M.: Phys. Rev. Lett. 81, 5912-5915 (1998)

14. Mali M., Roos J., Keller H., Conder K., Karpinski J.: Annu. Rep., pp. 56 and 57. Zürich: PhysikInstitut, University of Zürich 2001.

15. Williams G.V.M., Tallon J.L., Quitly J.W., Trodahl H.J., Flower N.E.: Phys. Rev. Lett. 80, 377380 (1998)

16. Lombardi A., Mali M., Roos J., Brinkmann D., Yakubowskii A.: Physica C 235-240, 1651-1652 (1994)

17. Yakubowskii A., Egorov A., Lütgemeier H.: Appl. Magn. Reson. 3, 665-676 (1992)

18. Suter A., Mali M., Roos J., Brinkmann D.: Phys. Rev. B 56, 5542-5551 (1997)

19. Zha Y., Barzykin V., Pines D.: Phys. Rev. B 54, 7561-7574 (1996)

20. Suter A., Mali M., Roos J., Brinkmann D.: Phys. Rev. Lett. 84, 4938-4941 (2000)

21. Suter A., Mali M., Roos J., Brinkmann D.: J. Magn. Reson. 143, 266-273 (2000)

22. Kitaoka Y., Hiramatsu S., Kohori Y., Ishida K., Kondo T., Shibai H., Asayama K., Takagi H., Uchida S., Iwabuchi H., Tanaka S.: Physica C 153-155, 83-86 (1988)

23. Takigawa M., Smith J.L., Hults W.L.: Phys. Rev. B 44, 7764-7767 (1991)

24. Mali M., Roos J., Keller H., Dooglav A.V., Sakhratov Yu.A., Savinkov A.V.: J. Superconductivity 15, 511-515 (2002)

Author's address: Detlef Brinkmann, Physik-Institut, University of Zürich, Winterthurerstrasse 190, 8057 Zürich

E-mail: Detlef.Brinkmann@physik.unizh.ch 